\title{
JOURNAL
}

of Health Inequalities

\section{Generating health in New York City}

\author{
Sonia Y. Angell \\ Columbia University, Department of Medicine, Division of General Medicine, New York City, NY, USA
}

ADDRESS FOR CORRESPONDENCE: Prof. Sonia Y. Angell, Columbia University, Department of Medicine, Division of General Medicine, PH8 East Room 105, 630 West 168 ${ }^{\text {th }}$ St., New York City, NY, 10032, USA, e-mail: sya2105@cumc.columbia.edu

Noncommunicable diseases (NCDs) are the leading causes of death in the US and the world, and related health inequities are substantial. New York City (NYC) provides an example of how urban areas are in a unique position to model, evaluate, and rapidly disseminate effective approaches to address this public health priority.

NYC is home to 8.4 million people, of whom nearly $40 \%$ are foreign born. The majority of NYC residents are of minority race/ethnicity, and one in five lives below poverty-level income. In 2016, cancer and cardiovascular diseases were responsible for one half of all early deaths, and the rate of death from heart disease before age 65 years in Blacks was 1.9 times higher than in Whites.

The NYC Department of Health and Mental Hygiene aims to reduce NCD risk by introducing sustainable changes in the environment that make healthier choices easier choices across the city. Changes include making nutritious foods more accessible, physical activity more regular, and tobacco use less feasible. Examples of NYC initiatives designed to improve diet quality include: the nation's first regulatory restriction on the use of trans fat in restaurants; warnings on high-sodium menu items in chain restaurants; the establishment of nutrition criteria for all foods purchased by NYC government; and the National Salt Reduction Initiative, to reduce sodium in packaged and restaurant foods, which has now expanded to address added sugar in packaged foods (NSSRI).

Evaluation of NYC's programs has demonstrated impact. For example, the total trans and saturated fat content of French fries sold in major chain restaurant went down $50 \%$ after the City's trans fat use restrictions went into effect. In a representative sample of NYC adults, after policy introduction, those who ate out frequently had a greater reduction in total serum trans fatty acids than those who ate out infrequently.
A central ethos of NYC's NCD approach has been to learn and work with other local, city, state, and global partners. The NSSRI was based upon a model first launched in the UK. The NSSRI now exists as a partnership of over 100 US state and local health authorities, and organisations. Take the Pressure Off, NYC!, a recently launched citywide hypertension initiative, includes over 120 organisations, representing 13 different sectors, united to address the problem of hypertension and related health disparities. The city's restaurant trans fat and sodium warning regulations, and government food procurement policies have served as models for other jurisdictions across the US and the world.

To reduce NCD prevalence, environments that make adopting health promoting behaviours must become the norm. Towards this end, cities can be key sites of innovation, demonstrating effective approaches to reduce health inequities and improve health across our communities.

Key words: healthy environment, noncommunicable diseases, health inequities, nutrition, prevention strategies.

\section{DISCLOSURE}

The author reports no conflict of interest. 